\title{
3 Research Square \\ Potential Prognostic Roles of Serum Lactate and Creatine Kinase Levels in Poisoned Patients
}

\author{
Alireza Golaghaei \\ Aja University of Medical Sciences \\ Hossein Hassanian-Moghaddam \\ SBMU \\ Shahin Shadnia \\ SBMU \\ NASIM ZAMANI ( $\square$ nasim.zamani@gmail.com ) \\ SBMU https://orcid.org/0000-0002-2091-0197 \\ Fatemeh Amraei \\ Zahedan University of Medical Sciences
}

Research article

Keywords: lactate, Creatine kinase, poisoning, prognostic factor

Posted Date: March 23rd, 2020

DOI: https://doi.org/10.21203/rs.2.20613/v2

License: (c) (i) This work is licensed under a Creative Commons Attribution 4.0 International License.

Read Full License 


\section{Abstract}

Background Examination of serum lactate Level and its changes, as an indicator of tissue oxygenation, as well as level of creatine kinase (CK) inhibitors, as a factor of mortality which partially expresses heart, brain, and muscle damage, may be considered as tools to determine prognosis in critically ill patients. We aimed to evaluate these two factors as potential prognostic factors in critically poisoned patients admitted to our toxicology ICU. Method: This is a cross-sectional descriptive-analytic study that was performed on poisoned patients referred to emergency department of Loghman Hakim Hospital. Onehundred critically poisoned patients who had been admitted to ICU were conveniently chosen using a random number table and included into the study after obtaining consent forms form their next of kin. Their serum lactate and CK levels were checked on admission. These levels were compared subsequently between survivors and non-survivors to seek for their potential prognostic role. Results: In a total of 100 patients enrolled, 61 were male. Serum level of lactate (with a cut off of $26 \mathrm{mg} / \mathrm{dL}$ ) and serum CK with a cutoff point of $169 \mathrm{U} / \mathrm{L}$ could have prognosticated death with sensitivity and specificity of $78 \%$ and $77 \%$ (for lactate) and $74 \%$ and $62 \%$ (for serum CK), respectively. Conclusions: In poisoned patients, serum lactate and $\mathrm{CK}$ can be used as possible prognostic factors because they rapidly increase in the serum and are easily detectable.

\section{Background}

Determination of the poisoned patients' prognosis since admission is an important factor that should always be considered by clinical toxicologists because of the limited intensive care unit (ICU) beds and nurses $[1,2]$.

In Iran, almost 650 people die annually due to poisoning and its complications. Although the pattern and trend of poisoning is different all over this country, the most common drugs causing

poisoning include sedatives, acetaminophen, and cardiologic drugs [3]. This is while there is limited access to professional health care personnel and facilities for intoxicated patients and this emphasizes the fact that these patients need to be prognostically evaluated since admission in order that those who would best benefit from specific care can be prioritized [4]. Although mortality of poisoning depends on the type of poisoning, early diagnosis and authentic treatment can be lifesaving [3].

Serum levels of lactate and creatine kinase (CK) have been used as possible prognostic factors in critical patients [5,6]. although studies on poisoned patients are not sufficient. Serum lactate is a potentially useful biomarker to risk-stratify patients with severe sepsis. However, it is plausible that elevated serum lactate is simply a manifestation of clinically apparent organ dysfunction and/or shock [6]. On the other side, serum CK can represent muscle, heart, and brain injuries. Measuring serum level of this enzyme is playing an important role in determination of severity of toxicity in patient at the risk of vital organs damage [7]. 
Since data in this regard is lacking in poisoned patients, we aimed to evaluate the potential role of serum lactate and CK as possible prognostic factors in 100 critically poisoned patients referred to a clinical toxicology tertiary center who were admitted to toxicology ICU due to severe poisoning.

\section{Methods}

In a cross-sectional descriptive study, allpatients who referred to poisoning emergency department of Loghman Hakim hospital between January 2016 and December 2017 were considered to be enrolled into the study. They were from those with confirmed poisoning and need to be admitted to ICU due to severity of poisoning (need for intubation, invasive treatments, and maintenance of antidotes) [2]. On the other hand, those whose treatment had begun before admission (referred from other centers), poisonings with critically increasing levels of lactate as a nature of it (including biguanids and anticonvulsants), and those whose next of kin did not consent to be included, were excluded from the study.

One-hundred critically ill patients admitted to toxicology ICU were conveniently chosen and enrolled into the study using a random number table. Blood samples were taken and serum lactate and CK levels were determined using spectrophotometry in the laboratory. A questionnaire which was designed by our team was used to collect the patients' data. Information included patients' demographic characteristics, on admission vital signs and lab tests, primacy CK and lactate, final diagnosis, hospital stay, and final outcome which was recorded for every single patient.

Statistical analysis was carried out using statistical package for social software (SPSS) software version 25. Mean (SD) and frequency (\%) of the data were used to describe the study population. Unpaired student's t-test was used to compare outcomes (survival versus death). To determine cut off point for serum lactate and CK, ROC curves were used.

Shahid Beheshti University of Medical Sciences ethics committee reviewed and approved the study protocol (IR.SBMU.RETECH.REC.1397.428). Oral consent was taken from all patients' next of keen before recruitment into the study.

\section{Results}

Of 100 patients enrolled into the study, 61 were male with a mean age of $34.01 \pm 14.65$ years and 39 were female with a mean age of $40.6 \pm 7.49$ years. Sixty-five patients survived. Mean hospitalization period was $2.69 \pm 2.12$ and $94.7 \pm 40.4$ hours in non-survivors and survivors, respectively. The most common cause of poisoning was drug overdose in 78 patients.

Basic characteristics and on-presentation vitals as well as biochemistry lab tests, liver function tests, and coagulation tests were similar between the two groups of survivors and non-survivors. Treatments given (intubation, inotropes, etc) were also similar between the groups. Mean serum lactate and CK were $38.1 \pm 2.4$ (range; 11 to 69 ) $\mathrm{mg} / \mathrm{dL}$ and $760.0 \pm 165.3$ (range; 57 to 3420 ) $\mathrm{U} / \mathrm{L}$ in non-survivors versus $22.3 \pm 0.7$ (range; 12 to 58 ) $\mathrm{mg} / \mathrm{dL}$ and $224.1 \pm 32.8$ (range; 33 to 1600 ) $\mathrm{U} / \mathrm{L}$ in survivors (Ps were $<0.001$ 
and 0.003), respectively. Spearman correlation of the study variables is shown in Table 1. Roc curves shown in Figures 1 and 2 were used to obtain cut-offs at which the prognosis could best be determined although this was not possible since AUC was less than the significant level. AUC was 0.859 (cut-off point of $26.50 \pm 0.045$ with sensitivity of $78 \%$ and specificity of $77 \%$ ) and 0.735 (cut-off point of $169.50 \pm$ 0.056 with sensitivity of $74 \%$ and specificity of $62 \%$ ) for lactate and CK, respectively.

\section{Discussion}

The prognostic and diagnostic utility of a serum lactate concentration in the initial evaluation of drug overdose is historically controversial. Lactate concentration is a useful prognostic indicator for mortality in both medical/surgical patients and undifferentiated ICU patients. Current guidelines for the initial approach to management of the patient with a drug overdose do not include routine evaluation of serum for a lactate concentration. However, lactate concentration is an established prognostic marker for the evaluation of patients with elevated anion gap metabolic acidosis, selected drug overdoses (metformin and acetaminophen), selected chronic drug toxicities (stadivudine), and chemical poisoning (aluminum phosphide and cyanide) [8-10].

As the report of national drug and poisoning information center (DPIC) of Iran provided, $60 \%$ of all contacts per year are related to poisoning [11]. Lactic acidosis is the condition where lactate concentration increase instantly to more than $5 \mathrm{mmol} / \mathrm{dL}$. Type $A$ of lactic acidosis occurs in oxygen distribution dysfunction due to hypotension or cyanosis [11]. Type B lactic acidosis occurs in sepsis, liver dysfunction, diabetes, and drugs such as biguanides, acetaminophen, and sorbitol [6,9].

Creatine kinase supplies energy in body organs with different types in brain (CK1), myocardium (CK2), and muscle (CK3) whose change is considered to be due to organ damages [5]. Usually, existence of CK in blood defines the organ injuries including myocardial infarctions, rhabdomyolysis, autoimmune myositis, and kidney injuries [7].

Our results are in accordance with those reported by Lee et al in paraquat-poisoned patients in early stages of poisoning [12] although they did not evaluate CK level. Manini and colleagues evaluated 50 cases and 100 controls among acutely-poisoned patients and determined that lactate was an excellent prognostic factor in poisoned patients [8]. They declared that using ROC analysis, initial venous lactate concentrations obtained in the ED had outstanding diagnostic test characteristics. By maximizing the sum of sensitivity and specificity, selection of optimal integer cut points for lactate concentration occurred at $3.0(27 \mathrm{mg} / \mathrm{dL})$ and $5.0 \mathrm{mmol} / \mathrm{L}$ [8] which is very close to our results (the most sensitive cutoff point of $26.5 \mathrm{mg} / \mathrm{dL}$ ).

Talbot and assistants defined cryptic shock as condition of shock in which blood pressure is normal but lactate is increased. They mentioned that hypoxia or toxins could destroy ATP since phosphorylation chain accelerates causing in accumulation of pyruvate and increased in Lactate level [13]. However, in a study on carbon monoxide-poisoned patients, although it was shown that serum lactate increases at the beginning of $\mathrm{CO}$ poisoning and this increase is compatible with patients' blood $\mathrm{CO}$ level and severity of 
neurologic symptoms, there was no statistically significant difference in neurological symptoms in study groups. This study concluded that serum lactate was not a beneficial criterion for intoxication determining (Spearman's test $r=0.3$ ) [14] that is not in line with our study. Mozaffari et al also mentioned that serum lactate was higher in patients who had died in toxicology ICU although their main focus was on ventilator-associated pneumonia and no direct correlation had been checked [15].

We also evaluated serum CK in accordance with serum lactate between survivors and non-survivors and tried to set a specific cut-off point to simplify evaluation of clinical condition of each patient and accelerate medical actions. Finally, lactate and CK level were higher in non-survivors with an acceptable cut-off point set to differentiate between these two group. It seems that Lactate and CK can be used in determination of prognosis in acute poisonings as Manini and colleagues had previously claimed [8].

\section{Conclusions}

Regarding acceptable results and easily available tests that are sensitive enough and increase quickly after the poisoning injury, serum lactate and CK can be used as potential prognostic factors in acutely poisoned patients. This can help us better manage these patients in over-crowded poisoning wards with limited ICU beds.

\section{Declarations}

\section{Ethics approval and consent to participate}

This study was approved by our local ethics committee in Shahid Beheshti University of Medical Sciences.

\section{Consent for publication}

Not applicable.

\section{Availability of data and materials}

The data that support the findings of this study are available from the corresponding author upon reasonable request.

\section{Competing interests}

The authors declare that they have no competing interests.

\section{Funding}

None.

\section{Authors' contributions}


AG and SS gave the idea. AG, NZ, and FA collected the data. HHM analyzed the data. NZ and FA drafted and finalized the manuscript. All approved the final manuscript.

\section{Acknowledgements}

None.

\section{Reference}

1. 1. Shadnia S, Zamani N, Hassanian-Moghaddam H, Shafaroodi $H$, Padandar M, Rezaeizadeh $M H$. Prognostic value of cortisol and thyroid function tests in poisoned patients admitted to toxicology ICU. World J Emerg Med. 2018;9(1):51-55.

2. Alizadeh AM, Hassanian-Moghaddam H, Shadnia S, Zamani N, Mehrpour O. Simplified acute physiology score II/acute physiology and chronic health evaluation II and prediction of the mortality and later development of complications in poisoned patients admitted to intensive care unit. Basic Clin Pharmacol Toxicol. 2014 Sep;115(3):297-300.

3. Samira Alinejad, Nasim Zamani, Mohammad Abdollahi, Omid Mehrpour, MD A Narrative Review of Acute Adult Poisoning in Iran. Iran J Med Sci. 2017 Jul; 42(4): 327-346.

4. Maurer $\mathrm{HH}^{1}$. How can analytical diagnostics in clinical toxicology be successfully performed today? Ther Drug Monit. 2012 Oct;34(5):561-4.

5. Zahraei Mahin, Ganjali Mohammad Hussein. Investigation of changes of enzyme creatine kinase and its isoenzyme on hypothyroidism. Journal of Tehran University of Medical Sciences.: 1990;43(1):55-58.

6. Mikkelsen ME, Miltiades AN, Gaieski DF, Goyal M, Fuchs BD, Shah CV, et al. Serum lactate is associated with mortality in severe sepsis independent of organ failure and shock. Critical care medicine. 2009;37(5):1670-7.

7. Pajoum A, Fahim F, Akhlaghdoust M, Zamani N, Amirfirooz Z, Dehdehasti M. Rhabdomyolysis and Acute Poisoning; a Brief Report. Emerg (Tehran). 2018;6(1):e56.

8. Manini AF, Kumar A, Olsen D, Vlahov D, Hoffman RS. Utility of serum lactate to predict drug-overdose fatality. Clin Toxicol (Phila). 2010 Aug;48(7):730-6.

9. Hasanshiri F, Pourabbasi M, Moosavi G, Fatahi M, Kianfar A, Seyedi H, et al. Association between serum lactate and postoperative outcomes following coronary artery bypass graft surgery. Feyz Journal of Kashan University of Medical Sciences. 2017;20.

10. Hashemi-Domeneh B, Zamani N, Hassanian-Moghaddam H, Rahimi M, Shadnia S, Erfantalab P, Ostadi A. A review of aluminium phosphide poisoning and a flowchart to treat it. Arh Hig Rada Toksikol. 2016 Sep 1;67(3):183-193.

11. Erfantalab P, Soltaninejad K, Shadnia S, Zamani N, Hassanian-Moghaddam H, Mahdavinejad A, Damaneh $\mathrm{BH}$. Trend of blood lactate level in acute aluminum phosphide poisoning. World $\mathrm{J}$ Emerg Med. 2017;8(2):116-120. 
12. Younghwan Lee, Jun Ho Lee, Ae Jin Seong, Chong Kun Hong, Hae Jong Lee, Dong Hyuk Shin, Seong Youn Hwang. Arterial lactate as a predictor of mortality in emergency department patients with paraquat intoxication. Clinical Toxicology (2012), 50, 52-56. Copyright @ 2012 Informa Healthcare USA, Inc.ISSN: 1556-3650 print / 1556-9519 online.

13. Mullen M, Cerri G, Murray R, Talbot A, Sanseverino A, McCahill P, Mangolds V,n Volturo J, Darling C, Restuccia M. Use of point-of-care lactate in the prehospital aeromedical environment. Prehosp Disaster Med. 2014 Apr;29(2):200-3.

14. Benaissa ML, Mégarbane B, Borron SW, Baud FJ. Is elevated plasma lactate a useful marker in the evaluation of pure carbon monoxide poisoning? Intensive CareMed. 2003 Aug;29(8):1372-5.

15. Mozafari N, Mortazavi H S, Alinia T, Barari B, Talaie H. Is Serum Lactate Level a Prognostic Factor for the Incidence and Mortality of Ventilator-Associated Pneumonia Among Poisoned ICU-Admitted Patients? Iran Red Crescent Med J. 2017; 19(1):e43951.

\section{Table}

Table 1: Survey of Spearman's correlation between study variables.

\begin{tabular}{|c|c|c|c|c|c|}
\hline & CK & Lactate & Duration of hospitalization & Consequences & Sex \\
\hline CK & 1.000 & ${ }^{* 0.294}$ & ${ }^{* *} 0.297$ & ${ }^{* * 0.348}$ & -0.159 \\
\hline Lactate & $0.294^{*}$ & 1 & 0.133 & $*^{* *} 0.537$ & -0.148 \\
\hline Duration of hospitalization & $0.297^{* *}$ & 0.133 & 1 & $*^{* *} 0.503$ & -0.005 \\
\hline Consequences & $0.384^{* *}$ & ${ }^{* *} 0.537$ & & 1 & 0.015 \\
\hline Sex & -0.159 & -0.148 & -0.005 & 0.015 & 1 \\
\hline
\end{tabular}

\section{Figures}




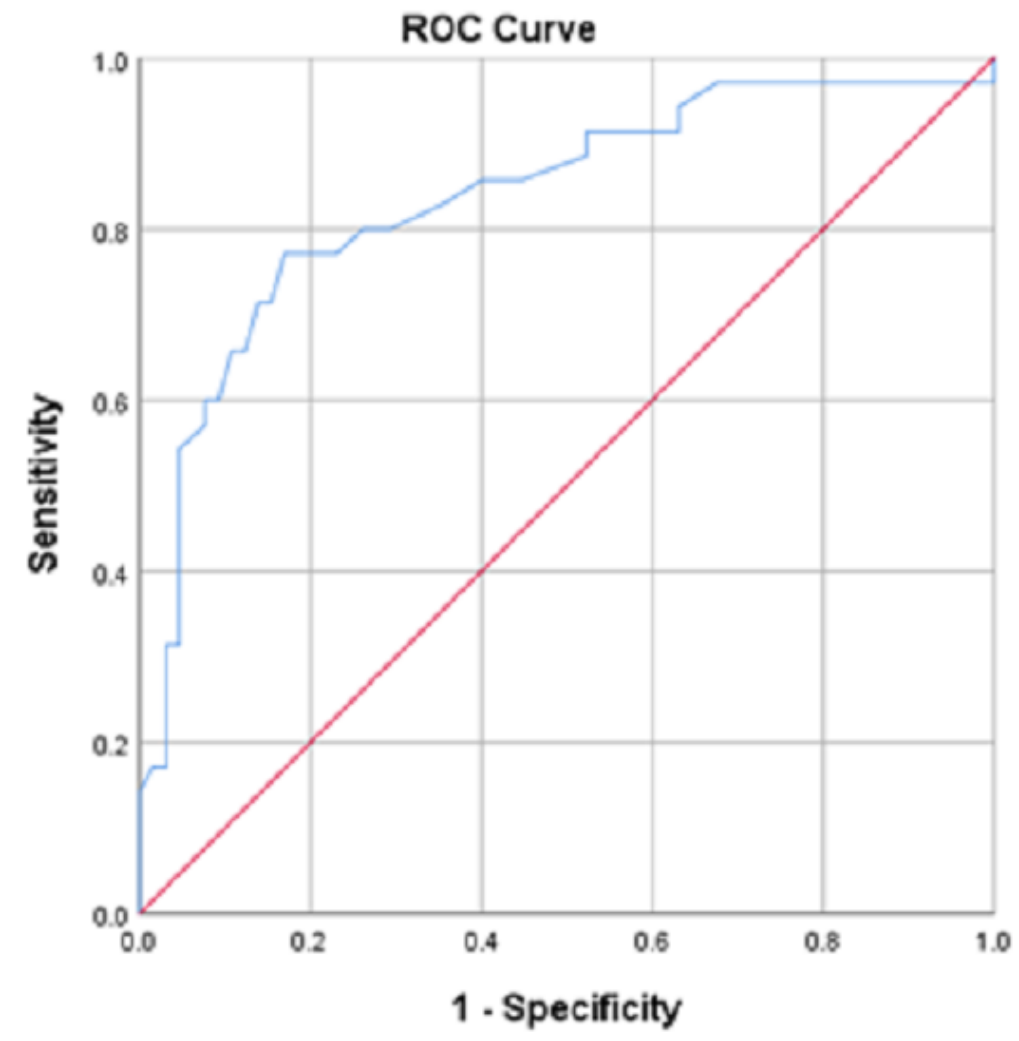

Diagonal segments are produced by ties.

Figure 1

ROC curve for serum Lactate level in dead patients 


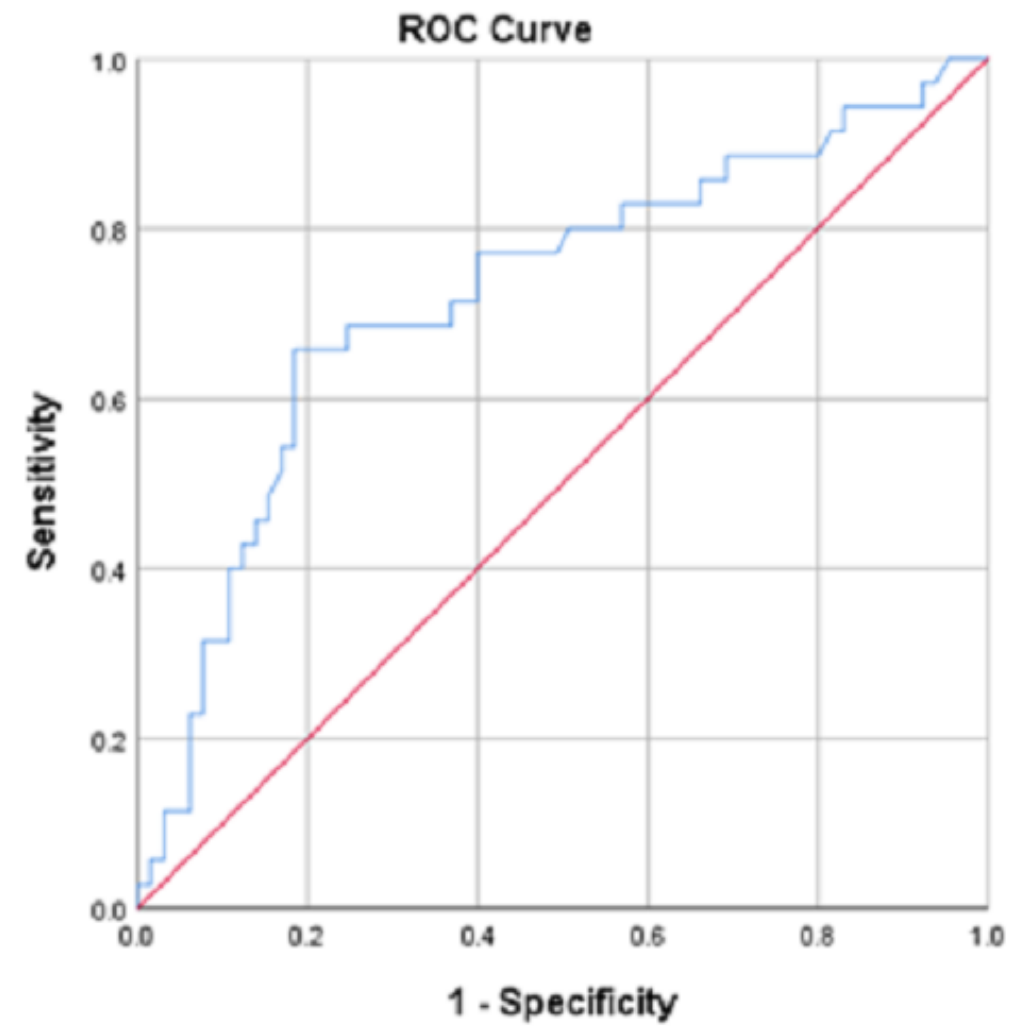

Diagonal segments are produced by ties.

Figure 2

ROC curve for serum CK level in dead patients 